\title{
Gasto social y desarrollo humano en el ámbito local. Evidencia de dos municipios del estado de Oaxaca, México
}

Ady P. Carrera-Hernández

\section{Resumen}

Desde finales de los 1990, México aumentó sus Programas de Transferencias Monetarias Condicionadas para los grupos más pobres; dando especial atención al desarrollo humano en áreas rurales. Esta investigación realizada en municipalidades indígenas del estado de Oaxaca, proporciona evidencia empírica sobre los factores que, además del diseño de las transferencias, determinan los resultados del gasto social para el desarrollo humano. Los hallazgos muestran consecuencias muy distintas en dos comunidades similares, lo que sugiere que a pesar de la inversión, si la capacidad de agencia de estas comunidades no se fortalece, los resultados no serán eficaces ni sostenibles. El trabajo aporta elementos para el debate acerca de la complejidad de reducir la pobreza en el nivel local en países menos desarrollados. Palabras clave: Municipios mexicanos, comunidades indígenas, desarrollo humano local, transferencias en efectivo condicionadas, sistema de usos y costumbres.

\section{Introducción}

El artículo se propone analizar los factores culturales, sociales y políticos que influyen en los resultados de los Programas de Transferencias Monetarias Condicionadas (PTMC) cuando se usan para promover desarrollo humano. ${ }^{22} \mathrm{El}$ texto se organiza en cinco secciones, la primera proporciona el contexto de los PTMC en América Latina. La segunda describe cómo los PTMC se han adoptado en México para promover el desarrollo humano; la tercera sección detalla el perfil de las comunidades indígenas del estado de Oaxaca y su particular forma de organización política. La cuarta analiza los estudios de caso de dos municipalidades indígenas oaxaqueñas a fin de aportar evidencia empírica sobre los factores que más allá del diseño técnico de las transferencias, determinan los resultados del financiamiento para el desarrollo humano. La última sección ofrece las reflexiones finales.

\section{Los Programas de Transferencias Monetarias Condicionadas (PTMC) en América Latina}

Los PTMC se adoptaron en la región durante la década de los 1990. A partir de entonces se han convertido en una de las herramientas principales de las políticas de combate a la pobreza. En 1997, tres países latinoamericanos los habían implementado; para 2010, estaban presentes en 18 de ellos. Estos programas crecieron no solo en número sino también en su monto, extendiéndose a otras regiones del mundo. De acuerdo con el Banco Mundial, para 2011, 300 países

\footnotetext{
${ }^{22}$ Conditional Cash Transfer Programmes, PTMC en ingles.
} 
tenían alguna forma de PTMC. $^{23}$ Muchos de esos programas se habían institucionalizado en distintos países con el apoyo de agencias multilaterales como el Banco Interamericano de Desarrollo (BID) y el Banco Mundial. Más allá de su efectividad, integralidad y principios, estos programas son considerados como un avance hacia sistemas sociales más amplios basados en valores de inclusión y universalidad (Cecchini \& Madariaga, 2011).

Los PTMC en América Latina otorgan efectivo a familias marginadas con la condición de que los niños asistan a la escuela y a consultas médicas de manera regular. Los objetivos de estos programas son dobles: invertir en asistencia social de corto plazo, mejorando el consume; y más importante aún, resolver pobreza futura mediante la formación y acumulación de capital humano (Rawlings \& Rubio, 2005). Teóricamente, las transferencias en efectivo presentan menos errores de inclusión que los subsidios porque entregan directamente el ingreso a la población objetivo. También evitan la creación de mercados secundarios y la distorsión de precios derivada de los subsidios; además que neutralizan las asimetrías de información ya que los beneficiarios escogen la mejor forma de gastar sus recursos para mejorar sus condiciones de vida de acuerdo con sus circunstancias específicas (Rowe, 2011).

Por otra parte, los PTMC construyen una relación directa entre el gobierno nacional y las familias con base en la corresponsabilidad para el uso apropiado de las transferencias. Muchos de estos programas seleccionan a las madres de familia la responsabilidad de manejar el dinero porque la evidencia internacional sugiere que ellas son mejores administradoras del ingreso de sus hogares cuando se trata del bienestar de sus hijos (Rawlings \& Rubio, 2005). El comportamiento individual no siempre se apega al óptimo social y existe el riesgo de que los recursos desincentiven a la gente de conseguir o conservar su empleo o de invertir en la acumulación de capital humano. Por lo tanto, puede lograrse algún tipo de equidad pero no de eficiencia. Por ello que es necesario establecer condiciones, para alinear el comportamiento individual lo más cerca posible al óptimo social (Rowe, 2011).

Los PTMC introdujeron un nuevo enfoque en la política social. Los programas tradicionales de asistencia se habían enfocado en mecanismos de transferencia para redistribuir el ingreso entre los más necesitados para ayudarlos a superar períodos cortos de pobreza durante tiempos de crisis (Rawlings \& Rubio, 2005; Brière \& Rawlings, 2006). Una de las características más positivas de los PTMC es su enfoque multidimensional acerca de la pobreza ya que nos solo se concentran en el ingreso sino también en educación, salud y nutrición (Rowe, 2011). Invertir en el capital humano de los grupos más pobres es una forma de estimular el ciclo virtuoso entre la protección social y el desarrollo humano. Además, como forma de inversión en 23

http://web.worldbank.org/WBSITE/EXTERNAL/TOPICS/EXTSOCIALPROTECTION/EXTSAFETY NETSANDTRANSFERS/0, contentMDK:20615138 menuPK:282766 pagePK:148956 piPK:2 16618 theSitePK:282761,00.html (08/03/2013) 
desarrollo y combate a la pobreza, estos programas son considerados la piedra angular para mejorar la política social y la gestión del gasto público. Esta forma de red de seguridad puede tener un rol estratégico para compensar las fallas del mercado que fomentan la pobreza, particularmente en países con gran inequidad (Brière \& Rawlings, 2006).

El primer programa de gran escala que combinó salud y educación fue Oportunidades en México, lanzado como Progresa en 1997. Con base en un modelo similar Colombia creó Familias en Acción; Honduras, el programa de Asistencia a la Familia; Jamaica, el programa de Avance a través de Salud y Educación; Nicaragua, el programa de Red Social de Protección; y Turquía, el Fondo de Solidaridad Social (Rawlings \& Rubio, 2005). Varios de estos programas han sido sistemáticamente evaluados para medir su efectividad e impacto. De acuerdo con el BID, basado en una rigurosa evaluación, los PTMC han tenido resultados positivos, como aumentar el consumo de comida y el calórico en la población de bajos ingresos, reduciendo la desnutrición crónica, particularmente en los niños menores de dos años; aumentado la asistencia diaria a la escuela y vigilando el crecimiento y las revisiones médicas preventivas; incrementando la matriculación en las escuelas y las tasas de avance, sobre todo en escuelas rurales y secundarias. ${ }^{24}$

La evaluación de la primera generación de estos programas en Colombia, México y Nicaragua "los presenta como medios efectivos para promover la acumulación de capital humano entre los hogares pobres. En particular, hay evidencia clara de su éxito en las tasas de matrícula escolar, cuidado preventivo de la salud y el aumento del consumo" (Rawlings \& Rubio, 2005:51). Además, los PTMC son considerados una forma de bajo costo para reducir la inequidad que puede ser replicada en muchos otros países. En Brasil y México, los PTMC fueron el segundo factor más importante en la reducción de la inequidad entre 1996 y 2004

Ya que los PTMC son valorados como una forma efectiva para promover el desarrollo humano, para los propósitos de este trabajo, es importante hacer una breve revisión de los componentes de dicho concepto. El desarrollo humano (DH) tiene dos aspectos: uno relacionado con el bienestar físico de la gente, lo cual incluye variables tales como salud, educación y nutrición; y el segundo está vinculado a la libertad, entendida como empoderamiento para tomar decisiones y disfrutar de participación política y ciudadana (Ranis y Stewart, 2000). Ambos aspectos están estrechamente vinculados, el primero se enfoca en construir capacidades y el segundo se refiere a la forma en que lo que la gente puede hacer con esas capacidades.

Amartya Sen llama al primero, el aspecto evaluativo del $\mathrm{DH}$, y al segundo el aspecto de agencia (Fukuda-Parr, 2003). El aspecto evaluativo puede ser medido con base en indicadores cuantitativos tales como un aumento en el ingreso,

24

$(28 / 02 / 2013)$

http://www.iadb.org/en/topics/social-protection/poverty-alleviation,1932.html 
expectativa de vida, años de educación escolar, nivel de nutrición, etc. El aspecto de agencia es mucho más difícil de medir ya que implica cambios políticos y sociales. Para alcanzar esta área del $\mathrm{DH}$ la gente debe actuar como un agente activo de su propio desarrollo, lo que requiere ser escuchado e involucrarse en la toma de decisiones que afectan a la comunidad (Agarwal, Humphries y Robeyns, 2003). Por lo tanto, para desarrollar su aspecto de agencia, las personas deben involucrarse en la acción colectiva con el fin de mejorar sus condiciones de vida y sus libertades políticas. Es por ello que algunos autores consideran que un componente esencial del $\mathrm{DH}$ es la participación ciudadana, el debate públicos y la toma de decisiones democráticas (Alkire y Deneulin, 2009).

Dada la importancia de los PTMC en las políticas sociales de los países latinoamericanos, es importante realizar un análisis más profundo de la forma en que funcionan y de los problemas que enfrentan cuando son implementados en contextos específicos con el fin de lograr un objetivo tan complejo como lo es el DH.

\section{PTMC y desarrollo humano en México}

Para mediados de la década de los 1990s, cerca de 30\% de la población total mexicana, y $50 \%$ de la población rural vivían en extrema pobreza. Paradójicamente, $75 \%$ del presupuesto total para consumo subsidiado estaba concentrado en áreas urbanas a pesar de que más de $60 \%$ de los pobres vivían en las rurales (Rowe, 2011:2). Progresa (Programa de Educación, Salud y Alimentación) fue lanzado en 1997 por el gobierno de Ernesto Zedillo. Progresa proporcionó transferencias en efectivo a los pobres rurales condicionando los recursos a que sus hijos asistieran a la escuela, visitas regulares para controles médicos y charlas sobre nutrición, salud y educación. Los PTMC reemplazaron varios programas basados en subsidios a la comida y otros tipos de transferencias en especie que mostraron ser ineficientes debido a razones políticas y logísticas. La evaluación general de Progresa fue muy exitosa para mejorar las condiciones de los más pobres (Gatner, 2007).

Cuando Vicente Fox ganó las elecciones presidenciales en 2000, su administración mantuvo el programa llamándolo Oportunidades, como es conocido actualmente. Éste ha sido el principal programa para promover $\mathrm{DH}$ entre las familias urbanas y rurales más pobres: "para lograr acceso a oportunidades iguales para vivir mejor, por lo tanto, logrando desarrollo humano y bienestar social para esta gente. El objetivo del programa es contribuir a romper el ciclo intergeneracional de pobreza, favoreciendo el desarrollo de capacidades en educación, salud y nutrición de las familias beneficiadas". ${ }^{25}$ El programa proporciona transferencias en efectivo a cambio de que asistencia regular a escuela y clínicas de salud; los pagos son entregados a las madres de familia o a la mujer que está al frente del hogar (Arnold \& Fuente, 2010).

25

http://www.oportunidades.gob.mx/Portal/wb/Web/oportunidades_a human_development_p rogram $(15 / 02 / 2013)$ 
La estrategia de DH en México tiene diversos programas y estrategias; en varios de ellos los gobiernos subnacionales tienen un rol importante. Es el caso de Estrategia $100 \times 100$ que fue creado en 2007. Su principal objetivo es estimular el desarrollo económico y social de las municipalidades con el más bajo grado de DH en el país. Aspira a aumentar el ingreso de la población, su productividad y oportunidades de trabajo; así como elevar sus estándares de vida mediante el acceso a mejores servicios de salud y educación, vivienda e infraestructura básica. ${ }^{26}$ Esta es una estrategia coordinada entre los gobiernos federal, estatales y municipales en seis áreas de inversión: educación, salud, productividad, vivienda, infraestructura y recursos naturales (cuadro 1). Su financiamiento proviene del presupuesto de cada uno de las 14 secretarías federales que participan y de los gobiernos estatales y municipales (CONEVAL, 2012). De acuerdo con las reglas del programa, el gobierno federal puede contribuir entre 60 y $100 \%$ del presupuesto total, además de las contribuciones de los estados y municipios, la sociedad, ONGs y beneficiarios también pueden contribuir con efectivo o donaciones en especie. Los gobiernos locales tienen que proporcionar el expediente técnico del proyecto para acceder a estos recursos. ${ }^{27}$

Cuadro 1. Áreas de inversión y acciones estratégicas del programa Estrategia $100 \times 100$

\begin{tabular}{|l|l|}
\hline Área & Acciones estratégicas \\
\hline Educación & Albergues, alfabetización, becas y escuelas. \\
\hline Infraestructura & $\begin{array}{l}\text { Agua potable, caminos, desarrollo urbano, energía eléctrica y } \\
\text { puentes. }\end{array}$ \\
\hline Ingreso & $\begin{array}{l}\text { Capacitación, ahorros, trabajos temporales y proyectos } \\
\text { productivos. }\end{array}$ \\
\hline $\begin{array}{l}\text { Medio } \\
\text { ambiente }\end{array}$ & $\begin{array}{l}\text { Cursos de medio ambiente, programas de conservación de la } \\
\text { naturaleza, proyectos ambientales, reciclaje, reforestación y } \\
\text { áreas naturales protegidas. }\end{array}$ \\
\hline Salud & $\begin{array}{l}\text { Adultos mayores, apoyo para comida, hospitales, clínicas } \\
\text { móviles y servicios médicos. }\end{array}$ \\
\hline Vivienda & $\begin{array}{l}\text { Estufas, paredes, pisos, habitaciones adicionales, } \\
\text { instalaciones de sanidad, techos y registro de la propiedad. }\end{array}$ \\
\hline
\end{tabular}

Fuente: CONEVAL, 2012:13

Desde 1997, el programa de Empleo Temporal (PET) es un apoyo para los períodos cortos de pobreza, contribuyendo a la protección social de la población afectada por la baja demanda de empleo o emergencias, proporcionando efectivo a hombres y mujeres mayores de 16 años. A cambio, ellos tienen que trabajar en proyectos que

${ }_{27}^{26}$ http://www.estrategia100x100.gob.mx/index.php?muest=2 (20/02/2013)

http://www.fenamm.org.mx/site/index.php?option=com content\&view=article\&id=750: estr ategia-100-x-100-busca-disminuir-los-altos-indices-de-pobreza-del-pais-diputados-asignan4900-mdp-para-beneficiar-hasta-125-municipios-\&catid=239 (22/02/2013) 
beneficien las condiciones de vida de las familias y comunidades. Este programa funciona en todo el país enfocado en municipalidades con muy alto, alto o mediano nivel de marginación o con alto nivel de desempleo. ${ }^{28}$ A partir de 2013 , de acuerdo con Cruzada por el Hambre, el programa creado por el nuevo gobierno federal, PET dará prioridad a la población y localidades que son objetivo de Cruzada (DOF, 2013). Los beneficiarios son seleccionados por la Secretaría de Desarrollo Social, y en ocasiones por las autoridades locales, quienes reciben los recursos, organizan y vigilan el trabajo de los beneficiarios.

Los resultados de los programas recién descritos son mixtos. Oportunidades beneficia a 6.5 millones de personas en todo el país, cubriendo especialmente a las municipalidades más marginadas. Proporciona apoyo a 187,000 localidades, $99 \%$ de la cuales son rurales y semi urbanas. ${ }^{29}$ En términos generales, diversas evaluaciones indican su impacto positivo en salud, educación y nutrición de los más pobres (Arnold \& Fuente, 2010). Aunque sus resultados en términos de la matrícula en escuelas primarias han sido insignificantes, ha tenido efectos positivos para disminuir las tasas de deserción. En el nivel de secundaria, el aumento ha sido sobresaliente, especialmente entre las chicas de áreas rurales. En el nivel de bachillerato, el incremento ha sido relevante, particularmente en las áreas rurales. En relación con los resultados en salud, la mayoría de los indicadores son positivos; los vinculados a pobreza actual señalan que el consumo ha aumentado elevando no solo los estándares de vida sino también garantizando un nivel básico de seguridad alimenticia. Sin embargo, los efectos sobre pobreza futura no pueden ser medidos aún, ya que los niños beneficiados desde 1997 apenas están terminando la escuela e integrándose a la fuerza de trabajo (Rowe, 2011).

Un estudio reciente del BID señala que a pesar de los buenos resultados obtenidos, algunos problemas han aparecido en los últimos años. Después de una década de crecimiento económico sostenido, los programas más grandes de la región latinoamericana, en Brasil, Colombia y México, han logrado una cobertura de cerca de 50 a $55 \%$ de los pobres; al mismo tiempo que la incidencia de la pobreza ha disminuido (Stampini \& Tornarolli, 2012:3). Una mayor cobertura significa que se han incluido más personas que no son pobres entre los beneficiarios. En México, la proporción de beneficiarios no pobres creció de 40 a $61 \%$ entre 2002 y 20120, y los jefes de familia de los hogares beneficiados permanecen en empleos informales (Ib.).

Estrategia $100 \times 100$ cubre 125 municipalidades en siete estados del país con 1.4 millones de beneficiarios (cuadro 2). En estas localidades, $84 \%$ de la población habla una lengua indígena. Los beneficiarios se distribuyen en 6,857 comunidades de las cuales $41 \%$ tienen menos de 50 habitantes, 231 más de mil, y sólo 13 tienen

${ }_{29}^{28}$ http://www.sedesol.gob.mx/es/SEDESOL/Mas informacion_del_Programa (10/02/2013)

http://www.oportunidades.gob.mx/Portal/wb/Web/oportunidades_a human_development_p rogram $(15 / 02 / 2013)$ 
más de 25 mil. $^{30}$ De 2007 a 2001, el presupuesto federal financio $87 \%$ de la inversión de este programa, y el área de infraestructura concentró el monto más grande de recursos, seguido muy lejos por el área de salud. La inversión ha sido inequitativa entre las áreas. Mientras que la inversión en infraestructura, ingreso y educación se redujo entre 2007 y 2011, salud, vivienda y recursos naturales aumentaron en el mismo periodo (CONEVAL, 2012:13-14).

Cuadro 2. Distribución de los beneficiarios de Estrategia $100 \times 100$ por estado

\begin{tabular}{|l||l||l|}
\hline Estado & Población & $\begin{array}{l}\text { Número } \\
\text { Municipalidades }\end{array}$ \\
\hline Chiapas & 423,023 & 20 \\
\hline Durango & 30,069 & 1 \\
\hline Guerrero & 373,908 & 21 \\
\hline Nayarit & 30,551 & 1 \\
\hline Oaxaca & 267,421 & 58 \\
\hline Puebla & 95,692 & 9 \\
\hline Veracruz & 181,216 & 15 \\
\hline Total & $1,401,880$ & 125 \\
\hline
\end{tabular}

Fuente: http://www.estrategia100x100.gob.mx/interna2.php (20/02/2013) La distribución entre municipios per cápita ha sido relativamente inequitativa. Entre 2007 y 2001, Mezquital, una municipalidad en el estado de Durango, recibió 8.7 veces más recursos que San Simón Zahuatlán en el estado de Oaxaca. Esto significa tres veces más que el resto de los 125 municipios cubiertos por $100 \times 100$ (Ib.). Esto podría deberse a que Durango tiene solo tiene un municipio en el programa y ello facilita la coordinación interinstitucional entre los niveles de gobierno y se focalizan mejor los recursos. Oaxaca es la entidad con las diferencias más grandes en inversión per cápita: mientras San Andrés Paxtlán recibió cerca de 218 dólares per cápita en 2008, San Martín Itunyoso obtuvo cerca de 1,056 dólares.

Esto se podría explicar porque Oaxaca es el estado con el mayor número de municipios en el programa, y por el impacto de la inversión en algunas localidades con población pequeña, como San Martín que solo tienen 2,554 habitantes (SEDESOL, 2008:42). La información disponible no es suficiente para determinar las razones específicas de esto, pero algunas explicaciones pueden ser: la región geográfica o económica, la densidad de población, el contexto político y cultural, y las dificultades para llegar a las comunidades lejanas (CONEVAL, 2012).

En relación a los resultados del PET, una evaluación reciente muestra que en $2011,70 \%$ de los beneficiarios percibieron mejoras en sus condiciones de vida y $80 \%$ mejoraron su ingreso. Sin embargo, los indicadores actuales no arrojan información confiable para determinar si este programa logra sus objetivos. Más aun, el PET tiene un problema de focalización ya que el término 'reducción temporal

${ }^{30}$ http://www.estrategia100x100.gob.mx/interna2.php (20/02/2013) 
del ingreso' no es explícito. EI PET aún requiere evidencia empírica para probar sus efectos positivos entre sus beneficiarios (Cejudo \& Michell, 2012).

Así pues, a pesar de sus diversos resultados positivos, los PTMC como cualquier otra herramienta de combate a la pobreza tiene limitaciones significativas. Considerados como 'balas mágicas en el desarrollo', estos programas han mejorado las políticas sociales pero no pueden ser considerados una panacea contra la

pobreza (Rowe, 2011). Los programas que aspiran a promover el desarrollo frecuentemente tienen efectos directos e indirectos no deseados. Los PTMC para las madres pueden desplazar otras transferencias privadas o afectar los incentivos para trabajar. También pueden perturbar las relaciones comunitarias si no todos los miembros reciben esos recursos (Rawlings \& Rubio, 2005). Su impacto también depende de la calidad y accesibilidad de los servicios que se supone deben mejorar (Brière \& Rawlings, 2006); y también de incentivos para la inversión y la creación de empleo (Rowe, 2011).

Con el fin de proveer evidencia acerca de la complejidad de usar los PTMC para promover el $\mathrm{DH}$ en el nivel municipal, la siguiente sección presenta el contexto de las comunidades indígena en Oaxaca, México; donde se localizan los dos casos de estudio analizados en este trabajo.

\section{Perfil de las comunidades indígenas en Oaxaca}

México es un país federal, por lo tanto, tiene tres ámbitos de gobierno: el federal, los estados y los municipios. Aunque está considerado como un país de ingreso medio, tiene uno de los niveles de inequidad más altos del mundo. Oaxaca es uno de los tres estados con los mayores niveles de analfabetismo (INEGI, 2011b). 41\% de la población no tiene acceso a servicios de salud (INEGI 2011a); 30\% no tiene agua potable, ni drenaje y sanitario en su hogar; y $6 \%$ no dispone de electricidad (INEGI, 2011a). Solo $21 \%$ tiene línea de teléfono, $40 \%$ cuenta con teléfono móvil y solo $8 \%$ tiene servicio de Internet (INEGI, 2011a).

La población económicamente activa de Oaxaca representa el 47\%; 32\% de ella trabaja en el sector primario, $20 \%$ en el secundario y $48 \%$ en el terciario (INEGI, 2011a). 70\% de la población vive con menos de nueve dólares al día (Hernández y Juan, 2007) y 67\% está por debajo de la línea de pobreza (CONEVAL, 2011). Esta breve descripción explica por qué Oaxaca es el estado con el mayor número de municipios en Estrategia $100 \times 100$ (cuadro 2). Estos tienen algunos de los niveles de DH más bajos del país. Hasta 2010, Oaxaca fue gobernada por el Partido Revolucionario Institucional (PRI) sin real competencia electoral. Sin embargo, ese año, ese partido fue derrotado por una coalición y el candidato de oposición ganó las elecciones para gobernador y ha estado en el poder desde 2011. Pero después de casi 80 años bajo el dominio del PRI, los actores políticos y la 
sociedad desarrollaron una cultura política basada en el clientelismo, paternalismo y el control de caciques (Audelo, 2007).

A pesar de su nivel de pobreza, Oaxaca tiene una ruca herencia étnica y cultural. Concentra el porcentaje más alto de población indígena del país. Hay 16 grupos etno lingüísticos de los 62 que existen en México (Blas, 2007:119), 34\% de su población de tres años en adelante habla una lengua indígena (INEGI, 2011a). El territorio de Oaxaca está dividido en 570 municipios que representan $23 \%$ del total del país. El sistema legal de Oaxaca fue el primero en reconocer el derecho de las comunidades indígenas para organizar su vida política con base en sus sistemas tradicionales, mejor conocidos hasta 2011, como Usos y Costumbres. ${ }^{31}$ (Armenta, 2006).

Anteriormente, las comunidades elegían sus autoridades locales con base en sus sistemas legales tradicionales y enviaban los resultados al gobierno estatal, que los registraba bajo el signo del PRI para que fueran legalmente reconocidos. De esta forma, el PRI mantenía su control político en todas las municipalidades (López, 2007; Audelo, 2007). A partir de 1995, dos sistemas electorales coexisten en el estado para elegir autoridades municipales: el sistema occidental, basado en la competencia de partidos políticos; y el de Usos y Costumbres (ahora conocido como Sistemas Normativos Internos), basado en las tradiciones de cada comunidad. A partir de entonces, 418 municipalidades eligieron sus gobiernos locales bajo el segundo sistema. ${ }^{32}$

Enfrentadas a la pobreza y el racismo, las comunidades indígenas han logrado sobrevivir con base en su trabajo comunitario que representa una forma tradicional de capital social. Tradicionalmente, las decisiones relativas a todos los asuntos de la comunidad son resueltas en asambleas comunitarias. La población realiza distintas actividades con el fin de mejorar las condiciones de vida de todos, desde construir un techo para la escuela primaria y limpiar los caminos, hasta desempeñarse como policía o autoridad municipal. Este servicio comunitario, conocido como Tequio, constituye la base de una especia de servicio civil que es obligatorio y que puede culminar con ser nombrado presidente municipal. Es por ello que las comunidades bajo el sistema de usos y costumbres han sido idealizadas como la forma perfecta de democracia (Cordero, 1999; Durand, 2007).

Sin embargo, el sistema no es homogéneo ya que cada comunidad tiene reglas distintas para elegir a sus autoridades y esto tiene implicaciones importantes para la forma en que sus habitantes interactúan. Lo cierto es que en varias de esas municipalidades, algunos grupos sociales están excluidos de la participación en las asambleas comunitarias y en la elección de autoridades locales. En algunas de ellas, las mujeres no pueden participar, en otras, los inmigrantes o la gente con religión

${ }^{31}$ A partir de agosto de 2011, este Sistema de elecciones locales se llama legalmente Sistemas Normativos Internos.

${ }^{32}$ En 2013, el congreso del estado de Oaxaca aprobó que uno de esos municipios cambiara al sistema de partidos políticos por lo que ahora son 417 . 
distinta a la católica, o que viven fuera de la cabecera municipal, no tienen el derecho de participar en la toma de decisiones o ser electas o votar por autoridades municipales.

Antes de 1990, había tres fuentes de conflicto en estas comunidades: i) intolerancia religiosa, ii) discriminación hacia las mujeres, y iii) violación de derechos humanos (Carlsen, 1999). En 1980, inició la descentralización del presupuesto federal y ello significó que las municipalidades empezaron a recibir recursos financieros, conocidos como Participaciones, por primera vez en su historia. En la década de los 1990s, se descentralizaron más fondos federales y aunque no son suficientes para las necesidades de los municipios, se han convertido en la principal fuente de conflicto entre los grupos locales, que en la mayoría de los casos son dirigidos por partidos políticos interesados en apoderarse de las regiones una vez monopolizadas por el PRI (Blas, 2007). La exclusión tradicional de grupos sociales y la lucha por el control de los recursos federales se han combinado, produciendo confrontación en muchas de estas comunidades, debilitando su capital social (Hernández-Díaz, 2007; Carrera \& Cadena, 2011; Carrera \& Cadena, 2012).

Estos problemas políticos y culturales son el contexto en el que las municipalidades indígenas reciben los PTMC y otros recursos para mejorar su nivel de DH. La siguiente sección presenta los estudios de caso de dos municipios indígenas de Oaxaca. Ellos proporcionan evidencia empírica sobre los factores que más allá del diseño técnico de las transferencias, determinan los resultados del financiamiento para el $\mathrm{DH}$.

\section{Casos de estudio}

\subsection{Santa Lucía Miahuatlán}

Localizado en la región Sierra Sur de Oaxaca, este municipio presenta altos niveles de marginación y no tiene ninguna actividad productiva que proporcione ingreso y empleo estable a su población. Ésta última es principalmente indígena y muchos de ellos no hablan español sino zapoteco, su lengua ancestral. En 2000, el Informe de Desarrollo Humano del Programa de las Naciones Unidas para el Desarrollo, lo ubicó entre los 10 municipios con el más bajo nivel del Índice de Desarrollo Humano (IDH) en el país. Pero pocos años después, la misma agencia lo colocó como el segundo de los 20 municipios de México con el mayor crecimiento de su IDH. Esto se debió a que aumentaron sus indicadores de salud, educación e ingreso (PNUD, 2008).

Esta comunidad se encuentra a 20 minutos de la ciudad que es el centro de la región Sierra Sur, y a dos horas y media de la capital del estado; hay servicio de taxis colectivos que conectan esta población con ambas ciudades. Tiene 3,356 habitantes y 723 hogares, de los cuales $80 \%$ tienen a un hombre como jefe de familia y 20\% a una mujer (INEGI, 2010). Santa Lucía está conformada por 11 pequeñas comunidades, incluyendo su cabecera municipal, la cual concentra al 50\% 
de la población total. La mayoría de esas comunidades albergan a menos de $10 \%$ de la población total y la provisión de servicios públicos es muy limitada o inexistente; entre otras razones por su geografía irregular y su dispersión. Algunas de las comunidades no están conectadas entre sí y carecen de infraestructura para ir de una a otra, en algunos casos se tiene que caminar hasta dos horas para llegar a alguna de ellas. En 2005, 99\% de la población mayor de cinco años, hablaba zapoteca, $73 \%$ también hablaba español y $23 \%$ hablaba solo zapoteca. Entre los que hablan solo zapoteca, $25 \%$ son hombres y $75 \%$ mujeres (INEGI, 2005). $86 \%$ de la población económicamente active labora en el sector primario, en cultivos de autoconsumo.

El municipio se rige por el llamado sistema de usos y costumbres y aunque todos los ciudadanos tienen el derecho de participar en la asamblea comunitaria para elegir a sus autoridades, la distancia entre las comunidades y el centro los detiene para participar. Lo que sucede entonces es que cada pequeña comunidad elige a sus representantes, quienes son los encargados de llevar sus demandas al ayuntamiento electo en la cabecera municipal. Todos los representantes integran el Comité Municipal para el Desarrollo Rural Sustentable, que solo tiene miembros del género masculino, aunque hace 20 años las mujeres adquirieron el derecho de participar en las asambleas. Prácticamente todo el presupuesto de Santa Lucía proviene de las transferencias federales, lo que significa que el gobierno local depende de esos recursos para mejorar las condiciones de vida de su población.

El cuadro 3 muestra cómo se han mejorado los servicios públicos en los hogares entre 2005 y 2010. Sin embargo, cerca de la tercera parte de ellos aún carece de agua potable en casa, una quinta parte no tiene energía eléctrica y la mitad carece de drenaje. La dependencia del gobierno local de los recursos federales implica que la inversión para infraestructura proviene de esos fondos. De acuerdo con registros oficiales, ${ }^{33}$ la mayoría de esos recursos se canalizaron para agua potable, electrificación, drenaje, salud y educación; lo que explica el avance en esos servicios. Los indicadores de salud mejoraron porque la tasa de mortandad de madres y niños disminuyó. Ello se debió a que las mujeres asisten al centro de salud para parir en lugar de ir con las parteras tradicionales, quienes no tienen ninguna capacitación médica. En todo caso, a pesar del progreso, esos indicadores no son buenos si se les compara con la media nacional. En 2005, la proporción de mortandad materna en Santa Lucía fue 136.24, en comparación con la nacional de 63.40; la mortandad infantil en el municipio fue de 35.59, mientras que la media nacional fue 16.76 (Secretaría de Salud, 2005).

Cuadro 3. Cobertura de los servicios públicos en los hogares de Santa Lucía Miahutlán 2005-2010

\begin{tabular}{|l|l|l|l|}
\hline Servicios públicos & 2000 & 2005 & 2010 \\
\hline
\end{tabular}

${ }^{33}$ http://www.observatoriomunicipal.org.mx/index.php/asistente/base de datos (13/03/11); y datos obtenidos del SNIM: http://www.rami.gob.mx/snim/fichabasica.php?t=mun finanzas (13/03/11). 


\begin{tabular}{|l|l|l|l|l|l|l|}
\hline Agua potable & 1 & $0.18 \%$ & 400 & $68.25 \%$ & 515 & $71.2 \%$ \\
\hline Energía eléctrica & 149 & $26.9 \%$ & 351 & $59.8 \%$ & 604 & $83.5 \%$ \\
\hline Drenaje & 3 & $0.54 \%$ & 5 & $0.85 \%$ & 384 & $53.1 \%$ \\
\hline Total de hogares & 552 & & 586 & & 723 & \\
\hline
\end{tabular}

Fuentes: INEGI, disponible en:

$<$ http://www.inegi.org. $\mathrm{mx} /$ sistemas/mexicocifras/default.aspx?ent $=15>$ $(13 / 03 / 11)$,

and $<$ http://sc.inegi.org. $\mathrm{mx} /$ sistemas/cobdem/primeraentrada.do? $\mathrm{W}=57$ \&Backidhecho=

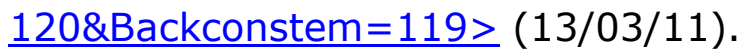

Los problemas culturales prevalecen porque las mujeres no quieren asistir con sus hijos a las consulta médicas preventivas. La mayoría de ellas son beneficiarias de Oportunidades por lo que están obligadas a hacerlo si desean conservar el apoyo económico. De acuerdo con personal del centro de salud, las mujeres aún no son conscientes de la importancia de la atención médica. Este centro de salud se construyó en 1979 con recursos federales y trabaja de 8 am a 5 pm. Después de estas horas, en caso de una emergencia, la gente tiene que trasladarse a la ciudad más cercana para recibir atención médica. En cada una de sus 11 comunidades hay una casa de salud bajo la responsabilidad de una persona que tiene conocimientos sobre primeros auxilios. El alcoholismo es un problema grave en este municipio, localizado en una región famosa por su mezcal; pero este producto no genera ningún beneficio económico para la población ya que ellos no lo producen. No obstante, sí estimula problemas sociales como violencia contra las mujeres e incluso asesinatos; pero ni el gobierno estatal ni el federal han adoptado medidas para tratar de solucionar esta situación. Hace pocos años, las autoridades municipales prohibieron el consumo de alcohol en las oficinas del ayuntamiento, lo que era una práctica común.

Santa Lucía tiene tres jardines de niños, dos de ellos se localizan en la cabecera municipal; cada una de las siete comunidades con mayor población tiene una escuela primaria y la única escuela secundaria de la población se encuentra en la cabecera municipal. Aunque la tasa de asistencia escolar mejoró entre 2000 y 2005, al final de este período, $11 \%$ de los niños entre 6 y 14 años de edad no asistían a la escuela, 93\% de las personas mayores de 15 años no habían completado la educación primaria y $45 \%$ de la población total era analfabeta, la mayoría de ella eran mujeres (CONAPO, 2005; INEGI, 2005). El aumento de los indicadores de educación podría ser resultado de los programas estatal y federal para alfabetización de adultos así como por las condiciones de Oportunidades.

El acceso al bachillerato es casi imposible ya que este nivel educativo no está disponible en el municipio; los estudiantes tienen que viajar a la ciudad más cercana y ello implica un costo extra. Además, los jóvenes muestran poco interés para continuar con su educación. A ello debe agregarse que $23 \%$ de la población solo habla su lengua nativa, $75 \%$ de ella son mujeres; lo que significa que ellas son aún 
más vulnerables que los hombres, con pocas oportunidades de acceder a la educación y por lo tanto, sin posibilidades reales de mejorar su ingreso. La gente entrevistada comentó que los maestros de sus escuelas son bilingües y por eso sus hijos no tienen que aprender español. Este no es solo un problema para los que no hablan español, incluso aquellos que son bilingües tienen un nivel muy básico de comunicación en español. Durante la visita a la comunidad fue necesario contar con alguien que actuara como traductor para conversar con algunas autoridades y con gente del municipio.

En 2005, Santa Lucía se ubicó entre los municipios con el más bajo ingreso en México, con impacto más negativo para las mujeres y los niños (PNUD, 2005:9). Pero se colocó entre las municipalidades mexicanas con el mayor incremento en su IDH, en parte porque su ingreso per cápita aumentó sustancialmente. Pero esto fue producto de que la mayoría de sus habitantes son beneficiarios de Oportunidades. Como se señaló, esta transferencia en efectivo tiene impacto en todos los indicadores de DH: Salud, consumo familiar, educación, asistencia permanente a la escuela e ingreso per cápita. El municipio también está incluido en Estrategia $100 \mathrm{x}$ 100 por su bajo IDH. Hasta 2011, 10 programas federales se habían adoptado en la comunidad a través de siete secretarías, cuya inversión tenía el propósito de reforzar el DH. Entre 2006 y 2010, la inversión federal en esta municipalidad se duplicó cada año.

Los indicadores revisados hasta ahora están relacionados con el aspecto evaluativo del $\mathrm{DH}$. Con el fin de tener una visión más integral es importante considerar el aspecto de agencia, el cual se vincula con el empoderamiento y la participación en la toma de decisiones. Con este propósito se conversó con dos grupos de mujeres, uno de la cabecera municipal y otro de la comunidad más lejana. El primer grupo dijo que tenían el derechos de participar en las asambleas comunitarias pero que no lo hacían por era 'una cosa de hombres'. El segundo grupo afirmó que ellas no participan porque sólo los hombres tienen el derecho de hacerlo. Entonces, la participación de las mujeres es muy limitada y ello constituye un enorme obstáculo para el desarrollo, como puede constatarse en las condiciones de marginación en las que se mantienen como el analfabetismo y monolingüismo. Los miembros del ayuntamiento siempre han sido hombres; fue hasta la administración 2008-2011 que una mujer joven fue aceptada en un puesto de apoyo debido a que contaba con estudios universitarios y ello era útil para las gestiones del municipio.

En este caso, Oportunidades ha afectado los incentivos de la gente para trabajar. Cuando se entrevistó al presidente municipal acerca de cómo resolver los problemas de su población, éste declaró que ellos no podían hacer nada, que era un asunto del gobierno, refiriéndose al gobierno federal, el cual debía mantener su apoyo. La mayoría de la gente entrevistada declare que debido a que recibían esta transferencia ya no buscaban trabajo estable. Una mujer dijo; "solía bordar blusas para ganar un poco de dinero pero el pago era muy bajo por un trabajo que lleva 
mucho tiempo. Ahora que recibo Oportunidades ya no lo hago más". Un hombre de una comunidad lejana comentó: "Ya no busco trabajo porque tengo Oportunidades". Algunas mujeres indicaron que aconsejan a otras mujeres más jóvenes para que tengan más hijos y así recibir más dinero.

A partir de agosto de 2009 , se empezó a aplicar un programa piloto en este municipio con el propósito de estimular el desarrollo rural sustentable y las capacidades individuales y colectivas locales para mejorar sus condiciones de vida a través de la planeación participativa. Este programa está enfocado en las actividades de las mujeres para mejorar la vida de sus familias y comunidades mediante el aprovechamiento de los recursos locales. Se tienen que organizar a sí mismas en grupos de trabajo que son liderados por una de ellas. El programa no ofrece ningún tipo de ayuda financiera o en especie, solo asesoría para los grupos. Una de las principales líderes de esos grupos expresó sus dudas acerca de la iniciativa porque las participantes no querían seguir trabajando ya que no recibían ningún tipo de ayuda material, ellas querían recibir algo a cambio de su participación. Un funcionario estatal comentó que este tipo de programas son muy paternalistas y no estimulan a las personas para desarrollar sus capacidades $y$ esfuerzos para superar la situación precaria en que viven.

\subsection{San Juan Mixtepec (Distrito 26) (34 $^{34}$}

Este es un municipio localizado en la misma región que Santa Lucía pero carece de las comunicaciones que tiene ésta última. Se requieren al menos dos horas para viajar de esta comunidad a la capital de la región, y cuatro horas para llegar a la capital del estado. Solo 28 de los 42 kilómetros entre San Juan y la capital regional están pavimentados y los derrumbes de las montañas son muy comunes en época de lluvias. El único transporte es el autobús municipal que sale cada madrugada a la capital regional y vuelve por la tarde. En 2010, San Juan tenía 688 habitantes que se concentraban en la cabecera municipal. 668 de ellos hablan zapoteco, 39\% son hombres y $61 \%$ mujeres. Este desequilibrio se debe a la migración de los varones a otras regiones del estado, otros estados e incluso a los EE UU. $80 \%$ de su población económicamente activa trabaja en el sector primario, el resto lo hace en el terciario como mercaderes o albañiles.

San Juan Mixtepec, como Santa Lucía, se ubicó entre los municipios mexicanos con el mayor aumento en si IDH en 2005. De acuerdo con el PNUD, esta municipalidad redujo su mortandad infantil, aumentó su tasa de alfabetismo y asistencia a la escuela; mejoró su nivel de salud e incrementó su ingreso per cápita; reduciendo su grado de marginación entre 2000 y 2005 (PNUD, 2008). Es una población pobre que no tiene ninguna actividad económica importante, por eso los hombres emigran en busca de mayor ingreso que el que les deja el cultivo del maíz

\footnotetext{
${ }^{34}$ Este número se usa para distinguir el municipio estudiado de otra comunidad con el mismo nombre que se localiza en la región de la Mixteca.
} 
y el frijol. El gobierno local tiene ingresos propios muy limitados por lo que depende de las transferencias federales. La mayoría de su población recibe distintos PTMC, el más importante de ellos es Oportunidades. El gobierno del estado también apoya los servicios de educación y salud, como ocurre en las demás municipalidades.

A diferencia del caso de Santa Lucía, San Juan solo tiene una comunidad, la cabecera municipal, en donde vive toda su población y su número de habitantes es muy pequeño. Sus autoridades locales también se eligen por el sistema de usos y costumbres en el que todos tienen el derecho de participar en la asamblea comunitaria para elegir al ayuntamiento. La asamblea toma todas las decisiones relacionadas con la vida del municipio, como la forma en que se invierten los fondos y las prioridades de obra pública. Como en la mayoría de este tipo de municipios, en San Juan existe el servicio comunitario llamado Tequio, que es considerado como una obligación moral para los miembros de la comunidad. La gente tiene que seguir un sistema de cargos vertical en el que empiezan como policías y pueden continuar hasta llegar a formar parte del ayuntamiento; de esta forma acumulan experiencia en la gestión municipal.

En 2010, 98\% de los hogares disponían de agua potable, 93\& de energía eléctrica y 45\& de drenaje (INAFED, 2010). Los recursos para financiar estos servicios vinieron básicamente de los programas federales pero el gobierno local proporcionó los fondos que le corresponden por ley y la población también contribuyó con trabajo y materiales locales. A las compañías que realizaron las obras se les solicitó que emplearan a albañiles del pueblo para beneficiar su economía. Hay una clínica médica que cuenta con una médico y una enfermera, quienes proporciona servicio las 24 horas del día durante todo el año, ya que viven en la propia clínica y así están disponibles en caso de emergencia. Esto ha ayudado a reducir la tasa de mortandad del municipio.

Antes de 2000, no había servicio médico y los que necesitan asistencia eran llevados a la capital de la región, muchos morían en el largo camino. La clínica fue construida en 2000 y se adquirió una moderna ambulancia equipada en 2008. Este vehículo se solicitó a la secretaría de salud del gobierno estatal, la cual pidió que el municipio pagara la mitad del costo. El ayuntamiento dio una parte de sus transferencias fiscales y la población también aportó dinero. Esta clínica cuenta con un salón de capacitación en donde se imparten pláticas educativas del programa Oportunidades acerca de salud, nutrición, control natal y enfermedades de transmisión sexual. De acuerdo con el personal, estas pláticas han generado cambios culturales positivos. Ahora las mujeres asisten a la clínica para parir en lugar de acudir con las matronas tradicionales, lo que ha reducido la tasa de mortandad materna e infantil. También han comprendido la importancia de las vacunas para mantener sanas a sus familias y de la limpieza regular en sus hogares y calles del pueblo, así como de la preservación del medio ambiente.

San Juan tiene servicios educativos desde el nivel de jardín de niños hasta bachillerato. El jardín de niños fue financiado con las transferencias fiscales que 
recibe el ayuntamiento de la federación; la escuela primaria la construyó el gobierno local y se amplió con base en el tequio de la población entre 1972 y 1973. La escuela secundaria se construyó en 1999, y la de bachillerato en 2000. Esta última es uno de los logros más importantes de esta población. Siendo un pueblo tan pequeño, no tenía el número de estudiantes necesarios para que el gobierno del estado construyera esta escuela, así que se les ofreció construirla en medio del camino entre San Juan y otro municipio vecino. Las autoridades de San Juan visitaron los municipios de los alrededores ofreciéndoles que si apoyaban su solicitud para construir el bachillerato en su municipio, San Juan ayudaría a sus estudiantes para asistir a esa escuela.

El bachillerato se inauguró en 2000 y las autoridades de San Juan cumplieron con su promesa. Proporcionan vivienda a los estudiantes de los demás municipios, quienes también comen en la cocina comunitaria del municipio por una pequeña cuota mensual. Algunos años, el número de estudiantes no ha alcanzado el mínimo requerido por el gobierno estatal para pagar los salarios de los profesores, así que el ayuntamiento de San Juan ha asumido ese costo. Incluso también han llegado a pagar los libros que se necesitan. El esfuerzo por mejorar el nivel educativo de la población ha rendido frutos, las generaciones más viejas no tuvieron otras opciones más que trabajar como albañiles o empleadas domésticas. Las nuevas generaciones logran empleos como asistentes en comercios o pequeños comerciantes. Toda la gente entrevistada manifestó su interés para que sus hijos estudien todos los niveles educativos con que cuenta la población porque es la única forma de mejorar su ingreso y de vivir mejor.

Otra situación sobresaliente de San Juan es que el español es el único idioma que se habla en las escuelas. La población lo decidió así hace varios años, ya que sus hijos necesitaban hablar español, para poder defender sus intereses y mejorar sus posibilidades de empleo. Actualmente, le número de personas que no hablan español es insignificante y son básicamente las personas ancianas que no tuvieron oportunidad de asistir a la escuela cuando eran jóvenes. Cuando se les preguntó acerca de la importancia de preservar su lengua ancestral dijeron que no es un problema ya que todo los hablan en casa y las asambleas se desarrollan en zapoteco. San Juan también ha buscado unir sus esfuerzos con los municipios vecinos para asfaltar los kilómetros que faltan del camino que los une a la capital regional. En 2007, se unió con otros nueve municipios para operar un módulo de maquinaria con el fin de dar mantenimiento a los caminos locales y mantener funcionando el que los lleva a la capital de la región. Antes de esto, en la época de lluvias, tenían que esperar la maquinaria federal o estatal, a veces por semanas sin comunicación con el resto de la región.

Cuando se entrevistó a antiguas y actuales autoridades del municipio, declararon que consideraban su cargo como una obligación moral para la comunidad aunque era una pérdida económica ya que no reciben salario. Las autoridades y la comunidad consideran que el progreso de su población está basado en la solidaridad 
y la confianza mutua. El capital social tradicional de este municipio se basa en la participación comunitaria que ha sido un factor clave para mejorar sus condiciones de vida. Las mujeres beneficiarias de Oportunidades que fueron entrevistadas fueron muy diferentes de las de Santa Lucía. Cuando se les invitó a platicar con el equipo de investigación, 50 de ellas llegaron a la clínica donde se desarrollaron las actividades. Todas ellas hablan muy bien el español y fueron muy abiertas para compartir los problemas de su comunidad. En general, dijeron que aunque el dinero que reciben es una suma pequeña, es útil para apoyar a sus hijos para que reciban una mejor educación.

Ninguna persona entrevistada dejo su trabajo por recibir esos recursos, por el contrario, las mujeres se quejaron de la falta de posibilidades para tener un empleo pagado en su pueblo. Expresaron su interés por abrir un taller para fabricar rebozos, cuya técnica ancestral está desapareciendo porque las nuevas generaciones no la quieren aprender. En años recientes, se han creado un par de proyectos productivos de piscicultura e invernaderos para el cultivo de jitomates.

\section{Reflexiones finales}

Los estudios de caso muestran que hay muchos factores culturales, sociales y políticos que influyen en los resultados de los PTMC en relación con el desarrollo humano. El caso de Santa Lucía representa un buen ejemplo de cómo esos programas pueden ser distorsionados. Es evidente que el avance de esta población en su IDH provino de los fondos masivos que el gobierno federal ha invertido debido a su clasificación como una de los municipios con más bajo nivel de DH en 2000. Mientras que el aspecto evaluativo del $\mathrm{DH}$ ha mejorado con base en la inversión en servicios públicos, no ha habido una estrategia para estimular el aspecto de agencia. La población de Santa Lucía está acostumbrada a políticas sociales basadas en el paternalismo debido al monopolio político del PRI de casi 80 años.

Es realmente difícil cambiar una cultura política tan arraigada, la que incluso a borrado la tradicional organización comunitaria que caracterizó a este tipo de municipios en el pasado. Los PTMC no han sido suficientes para modificar la forma en que esta población piensa acerca de su responsabilidad para promover su propio desarrollo. No han recibido ningún incentivo para reconstruir su capacidad de trabajo para mejorar su nivel de vida. La dispersión de las 11 comunidades que forman este municipio tampoco ayuda a que se conciban como un pueblo integrado que requiere del esfuerzo de todos para transformar sus condiciones de marginación. Las mujeres permanecen sin participar y las generaciones jóvenes no tienen estímulos para buscar mejores condiciones de vida. Los PTMC han reforzado el comportamiento conformista de la población y autoridades de Santa Lucía sin contribuir a desarrollar su libertad ni su participación política y ciudadana. No hay promoción de actividades productivas que proporcionen ingreso y autoempleo.

La evidencia de San Juan Mixtepec (Distrito 26) sugiere que la mejora de su IDH se ha basado en el esfuerzo de la comunidad y la confianza entre autoridades y 
población. Los valores comunitarios como la solidaridad y la unidad han permitido que esta remota comunidad vaya superando sus limitadas condiciones, demostrando que la sinergia entre el gobierno y la sociedad genera resultados positivos para el desarrollo. Una explicación de por qué esta comunidad ha conservado organización tradicional es precisamente que por estar tan aislada de todo, la única forma de sobrevivir fue a través del trabajo conjunto. Su tamaño pequeño y la concentración de su población en solo una comunidad la ha ayudado a mantener esta forma de trabajo. Además, también ha sido más fácil y barato proveer servicios públicos.

San Juan no ha recibido grandes montos de inversión federal, pero los que ha obtenido han sido administrados con gran eficiencia. No está incluido en Estrategia $100 \times 100$ pero ha logrado mucho mejores resultados en DH que Santa Lucía. En San Juan, los PTMC han apoyado el aspecto evaluativo del DH pero esto ha sido posible porque ya existía una base fuerte de capital social. De la misma forma, el aspecto de agencia ha sido reforzado por los PTMC a través de mejores niveles educativos para las mujeres y las nuevas generaciones. Estás últimas reconocen la necesidad de involucrarse en actividades productivas para que su progreso sea permanente. San Juan enfrenta los mismos retos que la mayoría de las comunidades indígenas: preservar sus tradiciones al mismo tiempo que se inserta en un mundo globalizado.

La evidencia presentada sugiere que el diseño de los PTMC y su implementación deben considerar cuidadosamente el contexto específico en el que se usarán. Como lo señalan Rawlings y Rubio: "no debe asumirse a partir de las resultados positivos de las evaluaciones de un pequeño grupo de países que puede obtenerse el mismo éxito en otros países en contextos distintos...tampoco los resultados positivos de un programa implican que éste es necesariamente el mejor enfoque para lograr un resultado en particular" (2005:52). Los resultados de Oportunidades en ambos casos arrojan luz acerca de la importancia de considerar que casi $20 \%$ de los municipios mexicanos son gobernados bajo el sistema de usos y costumbres. Esta condición requiere estrategias específicas para aprovechar la organización tradicional que puede ser encontrada en estas comunidades o recuperarla con incentivos adecuados.

El desarrollo humano no es fácil de lograr, particularmente su aspecto de agencia es realmente difícil de promover. Es por ello que debe considerarse que los PTMC deben ser parte de una estrategia más amplia para combatir la pobreza y promover el desarrollo humano, la cual requiere de un enfoque multidimensional. Lo casos recién analizados dan una idea acerca de la complejidad de impulsar el desarrollo humano. La consideración más importante puede ser que el propio esfuerzo de la gente es la piedra angular del proceso de desarrollo. Por lo tanto, "es claro que en ambientes de capacidades limitadas, es necesaria una mayor necesidad de apoyarse en las propias comunidades" (Brière \& Rawlings, 2006:4). La evidencia sugiere que no importa la cantidad de gasto social, si la capacidad de 
agencia de este tipo de comunidades no es reforzada, los resultados obtenidos con estos recursos no serán eficaces ni sostenibles.

\section{Fuentes consultadas}

Agarwal, Bina, Jane Humphries \& Ingrid Robeyns (2003), "Exploring the challenges of Amartya Sen's work and ideas: an introduction", en Feminist Economics, vol. 9, no. 2-3, pp. 3-12

Alkire, S. \& S. Deneulin (2009), "The Human Development and Capability Approach" en Deneulin, S. and L. Shahani (eds.), An Introduction to Human Development and Capability Approach, UK, Earthscan

Armenta R., Petra (2006), "Elecciones por usos y costumbres en México", en Revista Letras Jurídicas, vol. 14, Julio, pp. 1-10

Arnold, Margaret \& Alejandro de la Fuente (2010), Conditional cash transfer programs in Mexico: Progresa.Oportunidades, The United Nations Office for Disaster Risk Reduction, New York

Audelo C., Jorge (2007), "Organizaciones sociales y partidos políticos en Oaxaca: sus vínculos", en Política y Cultura, Primavera, no. 027, pp. 57-74

Blas López, Cuauhtémoc (2007), Oaxaca, ínsula de rezagos. Crítica a sus gobiernos de razón y de costumbre, Oaxaca, Editorial Siembra.

Brière, Bénédicte de la \& Laura B. Rawlings (2006), Examining Conditional Cash Transfer Programs: A Role for Increased Social Inclusion?, SP Discussion Paper no. 0603, World Bank Institute-Social Protection World Bank, Washington, D. C.

Carlsen, Laura (1999), "Autonomía indígena y usos y costumbres la innovación de la tradición", en Revista CHIAPAS, no. 7, disponible en: http://www.indigenas.bioetica.org/Carlsen.pdf (09/05/2010)

Carrera-Hernández, Ady P. y C. Cadena (2011), Democratización de políticas públicas para el desarrollo. La experiencia de municipios de usos y costumbres en Oaxaca, México, paper presented at XVI Congreso Internacional del CLAD sobre la Reforma del Estado y de la Administración Pública, Asunción-Paraguay

Carrera-Hernández, Ady P. y C. Cadena (2012), "Gestión municipal y desarrollo. La zona metropolitana de la ciudad Oaxaca", in Gretchen González (coord.), Reflexiones del desarrollo local sostenible, México, Universidad Autónoma Metropolitana Azcapotzalco, pp. 167-207

Cecchini, Simone \& Aldo Madariaga (2011), Programas de Transferencias Condicionadas. Balance de la experiencia reciente en América Latina y el Caribe, CEPAL, Santiago de Chile

Cejudo, Guillermo \& Cynthia Michell (2012), Meta evaluación del Programa de Empleo Temporal (PET), CIDE-CLEAR, México, available at: http://www.sedesol.gob.mx/work/models/SEDESOL/Resource/3158/1/images LPET MetaEvaluacion\%20.pdf 
CONEVAL. Consejo Nacional de Evaluación de la Política de Desarrollo Social (2012), Evaluación de impacto de la Estrategia 100 × 100, México

Cordero A., Carmen (1999), "Renovación de ayuntamientos en los municipios de elección por usos y costumbres", in José E. Ordóñez (Coord.), Pueblos indígenas y derechos étnicos. VII jornadas Lascasianas, IIJ, México, UNAM, pp. $107-114$

DOF. Diario Oficial de la Federación (2013), Acuerdo por el que los integrantes del Comité Técnico del Programa de Empleo Temporal emiten las Reglas de Operación del Programa de Empleo Temporal para el ejercicio fiscal 2013, jueves 28 de febrero, Primera Sección vespertina, México, pp. 1-39, available at:

http://www.sedesol.gob.mx/work/models/NORMATECA/Normateca/Reglas O peracion/2013/rop pet.pdf

Durand, Víctor (2007), "Prologo", en Jorge Hernández Díaz (coord.), Ciudadanías diferenciadas en un estado multicultural: los usos y costumbres en Oaxaca, México, UABJO-Siglo XXI, pp. 11-34

Fukuda-Parr, Sakiko (2003), "The Human Development Paradigm: Operationalizing Sen's Ideas on Capabilities", in Feminist Economics, vol. 9, nos. 2-3, pp. 301 $-317$

Gantner, Leigh (2007), PROGRESA: An Integrated Approach to Poverty Alleviation in Mexico, Cornell University, Ithaca, New York

Hernández Díaz Jorge (2007), "Dilemas en la construcción de ciudadanías diferenciadas en un espacio multicultural: el caso de Oaxaca", in Jorge Hernández Díaz (coord.), Ciudadanías diferenciadas en un estado multicultural: los usos y costumbres en Oaxaca, México, UABJO-Siglo XXI, pp. 35-86

Hernández Díaz, Jorge y Víctor L. Juan Martínez (2007), Dilemas de la institución municipal. Una incursión en la experiencia oaxaqueña, México, Miguel Ángel Porrúa-Universidad Autónoma Benito Juárez de Oaxaca

INEGI (2011a), Panorama sociodemográfico de Oaxaca, México, Instituto Nacional de Estadística y Geografía, disponible en: http://www.inegi.org. $m x /$ sistemas/biblioteca/detalle.aspx?c=28097\&upc $=702$ $\underline{825003362 \& s=e s t \& t g=330 \& f=2 \& p f=P o b \& e f=20 \& c l=0}$

INEGI (2011b), Principales resultados del Censo de Población y Vivienda 2010, México, Instituto Nacional de Estadística y Geografía

López, Clemente (2007), "Usos y costumbres y elecciones municipales en Oaxaca. La invención de un sistema electoral plural", en Centro de Estudios para la Transición Democrática, available at: http://www.cetrade.org/v2/revista transicion/2001/revista 34 federalismo y autonomias/lopez

Ranis, Gustav y Frances Stewart (2000), "Strategies for success in human development", in Journal of Human Development, vol. 1, no. 1, pp. 49-69 
Rawlings, Laura \& Gloria Rubio (2005), "Evaluating the Impact of Conditional Cash Transfer Programs", in The World Bank Research Observer, vol. 20, no. 1, Spring, pp. 30-55

Rowe, Nicholas (2011), Mexico's Oportunidades: Conditional Cash Transfers as the Solution of Global Poverty?, Keck Center for International \& Strategic Studies, USA

SEDESOL (2008), Reporte Anual 2008. Estrategia $100 \times 100$, Unidad de Microrregiones, México

Soares, Sergei, R. Guerreiro, F. Veras, M. Medeiros \& E. Zepeda (2009), "Conditional Cash Transfers in Brazil, Chile and Mexico: Impacts on Inequality", in Estudios Económicos, Número extraordinario, pp. 207-224

Stampini, Marco \& Leopoldo Tornarolli (2012), The growth of conditional cash transfers in Latin America and the Caribbean: did they go too far?, Policy Brief No. IDB-PB-185, IADB, 